University of Nebraska - Lincoln

DigitalCommons@University of Nebraska - Lincoln

Faculty Publications from the Department of Electrical \& Computer Engineering, Department Electrical and Computer Engineering

$11-15-1997$

\title{
Infrared optical properties of mixed-phase thin films studied by spectroscopic ellipsometry using boron nitride as an example
}

\author{
Mathias Schubert \\ University of Nebraska-Lincoln, mschubert4@unl.edu \\ B. Rheinlander \\ University of Leipzig \\ E. Franke \\ Institute of Surface Modification, Permoserstrasse 15, D-04303 Leipzig, Germany, efranke3@unl.edu \\ H. Neumann \\ Institute of Surface Modification, Permoserstrasse 15, D-04303 Leipzig, Germany \\ T. E. Tiwald \\ University of Nebraska-Lincoln \\ See next page for additional authors
}

Follow this and additional works at: https://digitalcommons.unl.edu/electricalengineeringfacpub

Part of the Electrical and Computer Engineering Commons

Schubert, Mathias; Rheinlander, B.; Franke, E.; Neumann, H.; Tiwald, T. E.; and Woollam, John A., "Infrared optical properties of mixed-phase thin films studied by spectroscopic ellipsometry using boron nitride as an example" (1997). Faculty Publications from the Department of Electrical and Computer Engineering. 43.

https://digitalcommons.unl.edu/electricalengineeringfacpub/43

This Article is brought to you for free and open access by the Electrical \& Computer Engineering, Department of at DigitalCommons@University of Nebraska - Lincoln. It has been accepted for inclusion in Faculty Publications from the Department of Electrical and Computer Engineering by an authorized administrator of DigitalCommons@University of Nebraska - Lincoln. 
Authors

Mathias Schubert, B. Rheinlander, E. Franke, H. Neumann, T. E. Tiwald, and John A. Woollam 


\title{
Infrared optical properties of mixed-phase thin films studied by spectroscopic ellipsometry using boron nitride as an example
}

\author{
M. Schubert* and B. Rheinländer \\ Faculty of Physics and Geoscience, University of Leipzig, Linnéstrasse 5, D-04103 Leipzig, Germany \\ E. Franke and H. Neumann \\ Institute of Surface Modification, Permoserstrasse 15, D-04303 Leipzig, Germany
}

T. E. Tiwald and J. A. Woollam

Center for Microelectronic and Optical Materials Research, and Department of Electrical Engineering, University of Nebraska-Lincoln, Lincoln, Nebraska 68588

J. Hahn and F. Richter

Institute of Physics, Faculty of Science, Technical University Chemnitz-Zwickau, D-09107 Chemnitz, Germany

(Received 9 December 1996; revised manuscript received 23 April 1997)

\begin{abstract}
We present a microstructure-dependent anisotropic infrared-optical dielectric function model for mixedphase polycrystalline material from which we derive the transverse and longitudinal-optical modes observable in thin films. Infrared ellipsometry over the wavelength range from 700 to $3000 \mathrm{~cm}^{-1}$ is then used to determine the phase and microstructure of polycrystalline and multilayered hexagonal and cubic boron nitride thin films deposited by magnetron sputtering onto (100) silicon. The ellipsometric data depend on the thin-film multilayer structure, the layer-phase composition, and the average orientation of the hexagonal grain $c$ axes. In particular, we demonstrate the existence of spectral shifts of longitudinal optical phonons as a function of microstructure, i.e., the average grain crystallographic orientation within the mixed-phase material. [S0163-1829(97)08244-1]
\end{abstract}

\section{INTRODUCTION}

Numerous investigations of the microstructure of polycrystalline and sometimes multilayered boron nitride (BN) thin films deposited by various chemical and physical vapor deposition methods ${ }^{1-3}$ have been reported using mainly high-resolution transmission electron microscopy (HRTEM) in combination with selected area electron diffraction (SAD). ${ }^{4-6}$ Hexagonal BN $\left(h\right.$-BN) has a layered $s p^{2}$-bonded graphitelike structure whereas cubic $\mathrm{BN}(c-\mathrm{BN})$ has an $s p^{3}$-bonded zinc-blende structure. The $h$-BN modification can be deposited in a nearly pure phase, with the texture of the $h$-BN grains depending on the growth conditions (e.g., substrate bias voltage $U_{B}$ ). This has been found recently for $\mathrm{BN}$ thin films deposited by magnetron sputtering from a study of the anisotropic polarized reflectance by generalized variable angle of incidence spectroscopic ellipsometry $\left(g\right.$ VASE) in the visible spectral range (VIS). ${ }^{7}$ High $c$-BN content thin films are known to consist of pure nanocrystalline $c$-BN grains (average size: $10-30 \mathrm{~nm}$ ) and $s p^{2}$-bonded $\left(h\right.$-BN) material at the grain boundaries. ${ }^{4}$ They are also deposited after the growth of a noncubic $\mathrm{BN}$ nucleation layer. ${ }^{5}$ This layer consists mainly of $h$-BN, and plays an important role in the $\mathrm{BN}$ film growth. A knowledge of phase and microstructure is a prerequisite for a better understanding and control of the thin-film growth and its physical properties.

Because of well separated $h-\mathrm{BN}$ and $c-\mathrm{BN}$ infrared lattice absorption frequencies, infrared-spectroscopy (IRS) has become widely used to determine the phase composition of $\mathrm{BN}$ thin films. ${ }^{1}$ Raman spectroscopy and IRS were applied to study the infrared optical properties of amorphous $(a-\mathrm{BN})$, turbostratic $(t-\mathrm{BN}), c-\mathrm{BN}$, and $h-\mathrm{BN} .^{8-11}$ Polarized IRS data are sensitive to the thin-film phase composition as demonstrated recently. ${ }^{11}$ However, the $h$-BN microstructure particularly affects the sample reflectivity behavior as well. ${ }^{12}$ The latter needs to be considered when analyzing infrared reflectivity data; otherwise it leads to ambiguous results.

The present paper focuses therefore on the application of infrared spectroscopic ellipsometry (IRSE) to simultaneously determine the phase and microstructure of BN thin films. We present a microstructure-dependent model for the $h$-BN infrared anisotropic dielectric function which allows us to analyze different anisotropic ellipsometric spectra observed on various multilayered $\mathrm{BN}$ thin films. The results obtained from the ellipsometric model are consistent with SAD and HRTEM investigations. We determine the amount of randomly oriented (isotropic) $h$-BN within the high $c$-BN content thin films from the infrared optical dielectric function using an effective medium approach. We obtain the infrared $\mathrm{BN}$ thin-film lattice resonance frequencies and average $c$-axis grain orientations in the layer from a detailed lineshape analysis of the IRSE data.

The object of this work - a simple macroscopic and nondestructive characterization of the phase and microstructure of $\mathrm{BN}$ thin films which is important for further development of this field-demonstrates the capability of spectroscopic ellipsometry to solve mixed-phase thin-film problems. We emphasize the method used here as an extension of infrared ellipsometry to polyphase and polycrystalline thin films. Whatever the ultimate object is from a technological point of view, i.e., whether to obtain pure and highly oriented phases, 
or to control mixed-phase samples, it will benefit from the analysis reported here. The theoretical considerations presented in Sec. III treat a rather ideal case of highly oriented material. However, more complicated distributions of grain orientations in mixed-phase layers can be described in a similar manner. Mixed-phase polycrystalline media are of general interest because they represent a large variety of physical properties of materials with constituents including pure-phase and single crystalline form.

Ellipsometry is a nondestructive optical technique which determines both phase and absolute value of the thin-film complex reflectance ratio $\rho$, defined as $\rho=R_{p} / R_{s}$ $=\tan \Psi \exp (i \Delta)$, where $R_{p}$ and $R_{s}$ are the reflection coefficients of light polarized parallel $(p)$ or perpendicular $(s)$ to the plane of incidence. ${ }^{13}$ The ellipsometric parameters $\Psi$ and $\Delta$ therefore contain information on both $p$ - and $s$-polarized light components reflected from the sample surface. In order to analyze the IRSE data, one needs to model the sample structure as well as the optical properties of the materials involved.

Several investigations of the infrared optical properties of $h$-BN and $c$-BN were reported on bulk or pyrolytic BN material. Geick, Perry, and Rupprecht ${ }^{14}$ found two characteristic $h$-BN lattice absorptions for electric-field polarizations $E$ parallel (out-of-plane, $\|$ ) and perpendicular (in-plane, $\perp$ ) to the lattice $c$ axis characterized by their transverse- (TO) and longitudinal- (LO) optical frequencies $\omega_{\mathrm{TO} \|}=783 \mathrm{~cm}^{-1}$, $\omega_{\mathrm{LO} \|}=828 \mathrm{~cm}^{-1}, \omega_{\mathrm{TO} \perp}=1367 \mathrm{~cm}^{-1}$, and $\omega_{\mathrm{LO} \perp}=1610$ $\mathrm{cm}^{-1}$, respectively. The large difference between the inplane and out-of-plane resonance vibration frequencies is indicative of the strong anisotropic optical behavior of bulk $h$-BN. Gielisse et al. ${ }^{15}$ estimated the characteristic $c$-BN lattice absorption (c) at the resonance frequencies $\omega_{\mathrm{TO}}=1065$ $\mathrm{cm}^{-1}$ and $\omega_{\mathrm{LO} c}=1340 \mathrm{~cm}^{-1}$, while Eremets et al. ${ }^{16}$ obtained $\omega_{\mathrm{TO} c}=1056 \mathrm{~cm}^{-1}$ and $\omega_{\mathrm{LO} c}=1305 \mathrm{~cm}^{-1}$.

\section{EXPERIMENT}

BN thin films were deposited on (100) silicon by reactive magnetron sputtering at various substrate bias voltages $U_{B}$, resulting in polycrystalline and nearly pure $h$-BN or $c$-BN thin films. Both rf magnetron sputtering from a $h$-BN target or a pure boron target, and dc sputtering from a pure boron target, were used for the BN thin-film deposition. ${ }^{17}$ With the boron target the process was reactive, where 10 -sccm nitrogen and $90-\mathrm{sccm}$ argon were sufficient for the growth of stoichiometric BN films. The gas flux during the process with the $h$-BN target was 3 -sccm nitrogen and $97-\mathrm{sccm}$ argon. The pressure was $0.2 \mathrm{~Pa}$ in all cases. The substrate temperature was $350^{\circ} \mathrm{C}$ during the deposition. The samples were investigated by HRTEM and SAD. ${ }^{18}$ Both high-content $c$-BN and $h$-BN thin films have a layered structure. Depending on the growth conditions, the $h$-BN layer grain $c$ axes show an average preferential orientation with respect to the film normal. ${ }^{7}$ This average orientation varies as a function of the growth condition $\left(U_{B}\right)$ as well. Some of the $h$-BN layers contain isotropic material with randomly oriented grain $c$ axes. The high $c$-BN content layers consist of a phase composite of pure $c$-BN and randomly oriented (isotropic) $h$-BN. ${ }^{12}$

Such samples have already been studied by $g$ VASE in the

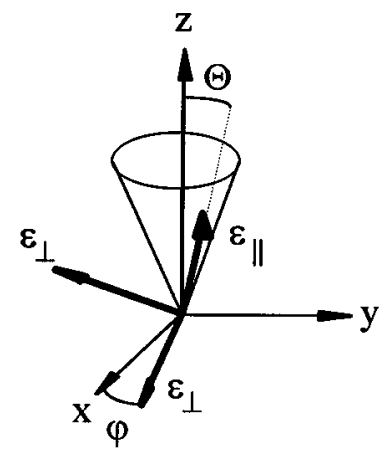

FIG. 1. Model of the geometry of the anisotropic $h$-BN thin-film microstructure from which we derive the dielectric function model used in this work. The microscopic $c$ axes are mapped onto the outer shell of a cone $(0 \leqslant \varphi \leqslant 2 \pi)$. The cone axis is the sample normal. The thin-film optical anisotropy depends on the average grain $c$-axes orientation $\Theta$. Note that fluctuations of grain $c$-axis orientations as well as more complicated angular distributions have not yet been accounted for in this model.

VIS spectral range, resulting in VIS optical constants. ${ }^{7}$ It was found that the $h$-BN thin films possess an effective optical axis perpendicular to the sample surface. The microscopic $c$ axes of the $h$-BN grains are more or less tilted by the same inclination angle $\Theta$ from the sample normal. The incident light beam then averages the grain inclinations over the range of in-plane orientations, thereby mapping the $c$ axes onto the outer shell of an effective cone (Fig. 1. Note that this model does not account for influence of angular distribution of grain $c$-axes). The cone angle $\Theta$ then describes the average orientation of the $c$ axes. The resulting $h$-BN dielectric function tensor was found experimentally to be independent of any in-plane sample orientation.

IRSE measurements were carried out in the wavenumber range between 700 and $3000 \mathrm{~cm}^{-1}$ at multiple angles of incidence $\left(\Phi_{a}=55^{\circ}-75^{\circ}\right)$. A rotating-polarizer, rotatingcompensator, Fourier-transform-based variable angle of incidence spectroscopic ellipsometer was used.

\section{RESULTS AND DISCUSSIONS}

Ellipsometric data were analyzed by modeling the thinfilm optical frequency dielectric function. According to Refs. 14 and 16, the complex dielectric function of bulk $h$-BN and $c$-BN within the reststrahlen region can be written as follows:

$$
\varepsilon_{j}=\varepsilon_{\infty j}+\frac{\left(\varepsilon_{0 j}-\varepsilon_{\infty j}\right) \omega_{\mathrm{TO} j}^{2}}{\omega_{\mathrm{TO} j}^{2}-\omega^{2}-i \omega \Gamma_{j}}
$$

where $\varepsilon_{0}$ is the static dielectric constant and $\varepsilon_{\infty}$ the highfrequency dielectric constant, $\omega_{\mathrm{TO}}$ is the transverse optical mode, $\Gamma$ is the resonance broadening, and $\omega$ is the incident light wave frequency. Note that all frequencies and broadening parameters are given in units of $\mathrm{cm}^{-1}$ throughout this work. The index $j$ refers to the $h$-BN in-plane " $\perp$ ", out-ofplane " "\|", and $c$-BN " $c$ " lattice absorption. We substitute $\varepsilon_{\infty}$ with $\omega_{\text {LO }}$ through the Lyddane-Sachs-Teller relation $\varepsilon_{\infty j}$ $=\varepsilon_{0 j}\left(\omega_{\mathrm{TO} j} / \omega_{\mathrm{LO} j}\right)^{2}$, where $\omega_{\mathrm{LO}}$ is the longitudinal-optical mode frequency. 


\section{A. $h$-BN dielectric function model}

Bulk $h$-BN is uniaxially negative $(a=2.504 \AA, c=6.661$ $\AA) .{ }^{19}$ The macroscopic dielectric function tensor of our polycrystalline $h$-BN thin films was found experimentally to be diagonal and independent of any in-plane sample orientation. Selected area electron-diffraction patterns from the same samples revealed an average preferential orientation of the $h$-BN grain $c$ axes with respect to the sample normal, but independent of the sample in-plane orientation. This result led to the assumption of the conelike distribution of the microscopic grain $c$-axis orientations. ${ }^{7}$ In this model the dielectric functions for polarization parallel $\left(\varepsilon_{e, \text { eff }}\right)$ and perpendicular $\left(\varepsilon_{o, \text { eff }}\right)$ to the thin-film normal then average between $\varepsilon_{\perp}$ and $\varepsilon_{\|}$according to Eqs. (2a) and (2b). They are obtained by integrating Eq. (12) in Ref. 20 over one period of the Euler angle $\varphi$ (the meanings of the symbols used there are now $\varepsilon_{0 x}=\varepsilon_{0 y}=\varepsilon_{\perp}$ and $\varepsilon_{0 z}=\varepsilon_{\|}$, where the cone angle $\Theta$ here is identical to the Euler angle $\Theta$ there, and $\psi$ is zero; see also Fig. 1):

$$
\begin{gathered}
\varepsilon_{o, \text { eff }}=\frac{1}{4}\left\{(3+\cos 2 \Theta) \varepsilon_{\perp}+(1-\cos 2 \Theta) \varepsilon_{\|}\right\}, \\
\varepsilon_{e, \text { eff }}=\varepsilon_{\|} \cos ^{2} \Theta+\varepsilon_{\perp} \sin ^{2} \Theta .
\end{gathered}
$$

Similar to the VIS spectral region (Eqs. (1) and (2) in Ref. 7) the infrared effective thin-film optical axis is perpendicular to the sample surface, and incident $p-(s-)$ polarized plane waves do not cause the exit of $s-(p-)$ polarized plane-wave components, respectively.

In the transverse optical-phonon mode, the ionic particle displacement is parallel to the thin-film boundary. ${ }^{21}$ The TO mode resonance frequencies for the $h$-BN thin films are obtained from the poles of $\varepsilon_{o \text {,eff }}$ given in Eq. (2a). Except for
$\Theta=0^{\circ}$, where only the in-plane TO mode $\omega_{\mathrm{TO} \perp}$ can be excited, two TO mode resonance frequencies are observable in $R_{p}$ and $R_{s}$ which are identical to both in-plane and out-ofplane TO modes, $\omega_{\mathrm{TO} \perp}$ and $\omega_{\mathrm{TO} \|}$, respectively. Note that if the broadening of the lattice resonance is considered as well, the apparent TO modes shift toward lower wave numbers (Ref. 22):

$$
\widetilde{\omega}_{\mathrm{TO}}=\omega_{\mathrm{TO}}\left[1-\left(\frac{\Gamma}{2 \omega_{\mathrm{TO}}}\right)^{2}\right]^{1 / 2} \text {. }
$$

However, $\widetilde{\omega}_{\mathrm{TO} \|}$ and $\widetilde{\omega}_{\mathrm{TO} \perp}$ are independent of the cone angle $\Theta$.

In the longitudinal-optical-phonon mode, the ionic particle displacement is perpendicular to the thin-film boundary. The LO-phonon mode frequency can be obtained from the dielectric medium properties at $\varepsilon_{e, \text { eff }}=0$. In bulk crystals, plane parallel electromagnetic waves do not couple to LOphonon modes. However, this situation changes for thin films with thicknesses less than the wavelength of the incident light. Plane waves with frequencies slightly below the LO mode attenuate but still propagate through the thin film. This is known as the Berreman-effect: At non-normal incidence the LO mode absorbs the incident $p$ polarization (i.e., plane electromagnetic waves with field components polarized parallel, as well as perpendicular, to the sample normal) leading to a characteristic signal in the $p$-polarized reflectivity $R_{p} \cdot{ }^{23}$

The $h$-BN thin-film LO modes $\widetilde{\omega}_{\mathrm{LO} \|}$ and $\widetilde{\omega}_{\mathrm{LO} \perp}$ depend on the cone angle $\Theta$, and can be calculated from the roots of Eq. (2b). For simplicity, and only in this derivation, we assume no broadening, i.e., $\Gamma_{\|}=0$, and $\Gamma_{\perp}=0$. From Eq. (2b), we then obtain

$$
\omega_{+/-}=\left(\frac{\alpha+\sqrt{\alpha^{2} \pm 4 \beta\left(\varepsilon_{0 \|} \cos ^{2} \Theta+\varepsilon_{0 \perp} \sin ^{2} \Theta\right) \omega_{\mathrm{LO} \|}^{2} \omega_{\mathrm{TO} \|}^{2} \omega_{\mathrm{LO} \perp}^{2} \omega_{\mathrm{TO} \perp}^{2}}}{2 \beta}\right)^{1 / 2},
$$

with

$$
\begin{gathered}
\alpha=\varepsilon_{0 \|} \omega_{\mathrm{LO} \perp \|}^{2} \omega_{\mathrm{TO} \|}^{2}\left(\omega_{\mathrm{LO} \|}^{2}+\omega_{\mathrm{TO} \perp}^{2}\right) \cos ^{2} \Theta \\
+\varepsilon_{0 \perp} \omega_{\mathrm{LO} \|}^{2} \omega_{\mathrm{TO} \perp}^{2}\left(\omega_{\mathrm{LO} \perp}^{2}+\omega_{\mathrm{TO} \|}^{2}\right) \sin ^{2} \Theta, \\
\beta=\varepsilon_{0 \|} \omega_{\mathrm{LO} \perp}^{2} \omega_{\mathrm{TO} \|}^{2} \cos ^{2} \Theta+\varepsilon_{0 \perp} \omega_{\mathrm{LO} \|}^{2} \omega_{\mathrm{TO} \perp}^{2} \sin ^{2} \Theta,
\end{gathered}
$$

where $\omega_{+}=\widetilde{\omega}_{\mathrm{LO} \perp}$ and $\omega_{-}=\widetilde{\omega}_{\mathrm{LO} \|}$. Hence, depending on the microstructure, i.e., the average $c$-axis grain orientation $\Theta$ within the $h$-BN thin film, the LO-phonon modes shift between $\omega_{\mathrm{LO} \|} \geqslant \widetilde{\omega}_{\mathrm{LO} \|}>\omega_{\mathrm{TO} \|}$ and $\omega_{\mathrm{TO} \perp}<\widetilde{\omega}_{\mathrm{LO} \perp} \leqslant \omega_{\mathrm{LO} \perp}$ as $\Theta$ varies from $0^{\circ}$ to $90^{\circ}$. Note that for $\Theta=0^{\circ}\left(90^{\circ}\right)$ the second LO mode disappears and only $\widetilde{\omega}_{\mathrm{LO} \|}\left(\widetilde{\omega}_{\mathrm{LO} \perp}\right)$ can be excited. Figure 2 depicts the thin-film LO modes as a function of $\Theta$. The bulk $h$-BN data from Ref. 14 were used for the calculations $\left(\Gamma_{\|}=\Gamma_{\perp}=0\right)$. Note the axis break and different scales for the in-plane and out-of-plane resonance spectral region. A distinct variation of the apparent LO modes $\widetilde{\omega}_{\mathrm{LO} \|}$ (open squares) and $\widetilde{\omega}_{\mathrm{LO} \perp}$ (open circles) is seen as the microstruc- ture of the $h$-BN thin films changes. As $\Theta$ tends toward $90^{\circ}$, the out-of-plane LO mode merges with $\omega_{\text {TO\| }}$ and disappears. Also, for $\Theta$ close to $0^{\circ}$, the in-plane LO mode $\widetilde{\omega}_{\mathrm{LO} \perp}$ converges toward $\omega_{\mathrm{TO} \perp}$. Figures 3(a) and 3(b) demonstrate the effect of the above-discussed infrared dielectric properties on the $h$-BN thin-film reflectivity by simulated $R_{p}$ and $R_{s}$ spectra. The same bulk data were used for the calculation (see Table I), and a uniaxial thin film $(d=300 \mathrm{~nm})$ was modeled through Eqs. (2a) and (2b) at a transparent half-infinite substrate (silicon, $\varepsilon=12.4$ ). The broadening parameters $\Gamma_{\perp}$ and $\Gamma_{\|}$were set to 60 and $40 \mathrm{~cm}^{-1}$, respectively, to account for the more realistic polycrystalline thin-film properties. The polarization-dependent reflectivity coefficients were then obtained from a $4 \times 4$ matrix algorithm which allows for arbitrary sample optical anisotropy. ${ }^{20}$ Figure 3(a) shows parts of the $p$ and $s$ reflectance versus wave number within the outof-plane resonance spectral region for various cone angles $\Theta$ and light incident at an angle of $\Phi_{a}=75^{\circ}$. Here the Berreman effect becomes obvious: the LO mode $\widetilde{\omega}_{\text {LO }}$ ab- 


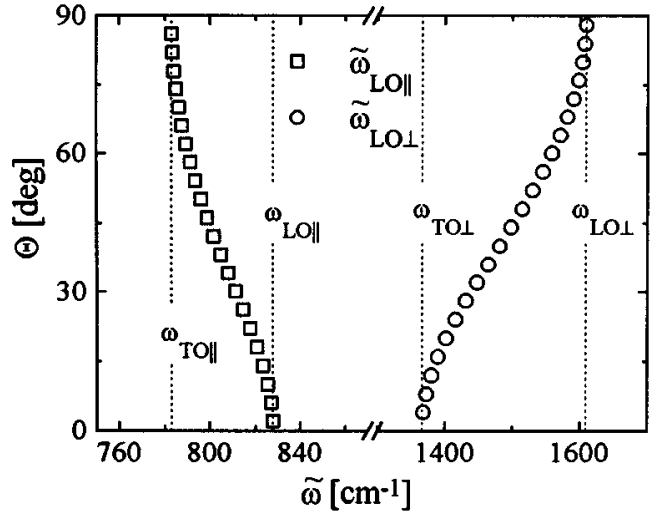

FIG. 2. Simulations of the longitudinal optical lattice resonance frequencies of polycrystalline $h$-BN thin films as a function of the average grain $c$-axis orientation $\Theta$. Except for $\Theta=0^{\circ}$ or $90^{\circ}$ both $\widetilde{\omega}_{\mathrm{LO} \|}$ (open squares) and $\widetilde{\omega}_{\mathrm{LO} \perp}$ (open circles) can be excited. The dotted lines indicate the bulk $h$-BN in-plane and out-of-plane TO and LO mode frequencies. The LO mode $\widetilde{\omega}_{\mathrm{LO} \|}\left(\widetilde{\omega}_{\mathrm{LO} \perp}\right)$ depends on $\Theta$ and converges to $\omega_{\mathrm{TO} \|}\left(\omega_{\mathrm{TO} \perp}\right)$ as $\Theta$ tends toward $90^{\circ}\left(0^{\circ}\right)$, where $\widetilde{\omega}_{\mathrm{LO} \|}\left(\widetilde{\omega}_{\mathrm{LO} \perp}\right)$ finally disappears, respectively. Bulk $h$-BN data $\left(\omega_{\mathrm{TO} \|}, \omega_{\mathrm{LO} \|}, \varepsilon_{0 \|}, \omega_{\mathrm{TO} \perp}, \omega_{\mathrm{LO} \perp}\right.$, and $\left.\varepsilon_{0 \perp}\right)$ were used for the calculations (Ref. 14). Note that no broadening was assumed, i.e., $\Gamma_{\|}$ $=0, \Gamma_{\perp}=0$.

sorbs the $p$-polarized wave components, increasing the reflected light intensity, whereas the signal in $R_{s}$ is only due to the TO mode $\omega_{\mathrm{TO} \|}$. The shift of the LO mode $\widetilde{\omega}_{\mathrm{LO} \|}$ as a function of $\Theta$ is indicated by arrows. For $c$-axis grain orientations nearly parallel to the thin-film boundary, $\widetilde{\omega}_{\mathrm{LO}}$ merges with $\widetilde{\omega}_{\mathrm{TO} \|}$ and disappears. The TO mode $\widetilde{\omega}_{\mathrm{TO} \|}$ is not affected by the average $c$-axis grain orientation, as mentioned above. As well, the TO mode vanishes for $\Theta=0^{\circ}$. Figure 3(b) presents parts of the same simulation for the in-plane resonance spectral region. Again, $\widetilde{\omega}_{\mathrm{LO} \perp}$ shifts as a function of $\Theta$ and merges with $\widetilde{\omega}_{\mathrm{TO} \perp}$ as $\Theta$ tends to small angles. At $\Theta=0^{\circ}$, only the TO mode is excited, representing the unusual case where the thin film reveals its LO mode at wave numbers less then the observable TO mode. This must be regarded as being due to the strong anisotropy of $h$-BN. Note also the asymmetric shape of both $R_{p}$ and $R_{s}$ which is due to the lattice resonance broadening.

The small shoulder in $R_{p}$ and $R_{s}$ below $\widetilde{\omega}_{\mathrm{TO} \perp}$ is due to an interference effect: both the real and imaginary parts of $\varepsilon_{o \text {,eff }}$ increase rapidly as $\omega$ tends toward $\widetilde{\omega}_{\mathrm{TO} \perp}$, which causes interference effects with diverging periods and asymptotic damping. As the thickness increases, these reflectivity oscillations become more pronounced, and may be observable if the lattice resonance broadening is small (see, e.g., the single crystal $c$-BN reflectivity data reported by Eremets et al., Fig. 9 in Ref. 16). The particular line shape of the reflectivity coefficients is a function of the layer thickness as well. The reader may refer to the calculated reflectance spectra of nonoriented polycrystalline $h$-BN thin films given by Friedmann et al. (Fig. 2 in Ref. 1). The reflectance extrema shift to higher wave numbers as the layer thickness increases. Also, the reflected light intensity changes significantly in the TO resonance frequency spectral range.

Because of their definition as the complex reflectance ratio, the ellipsometric parameters $\Psi$ and $\Delta$ contain spectral
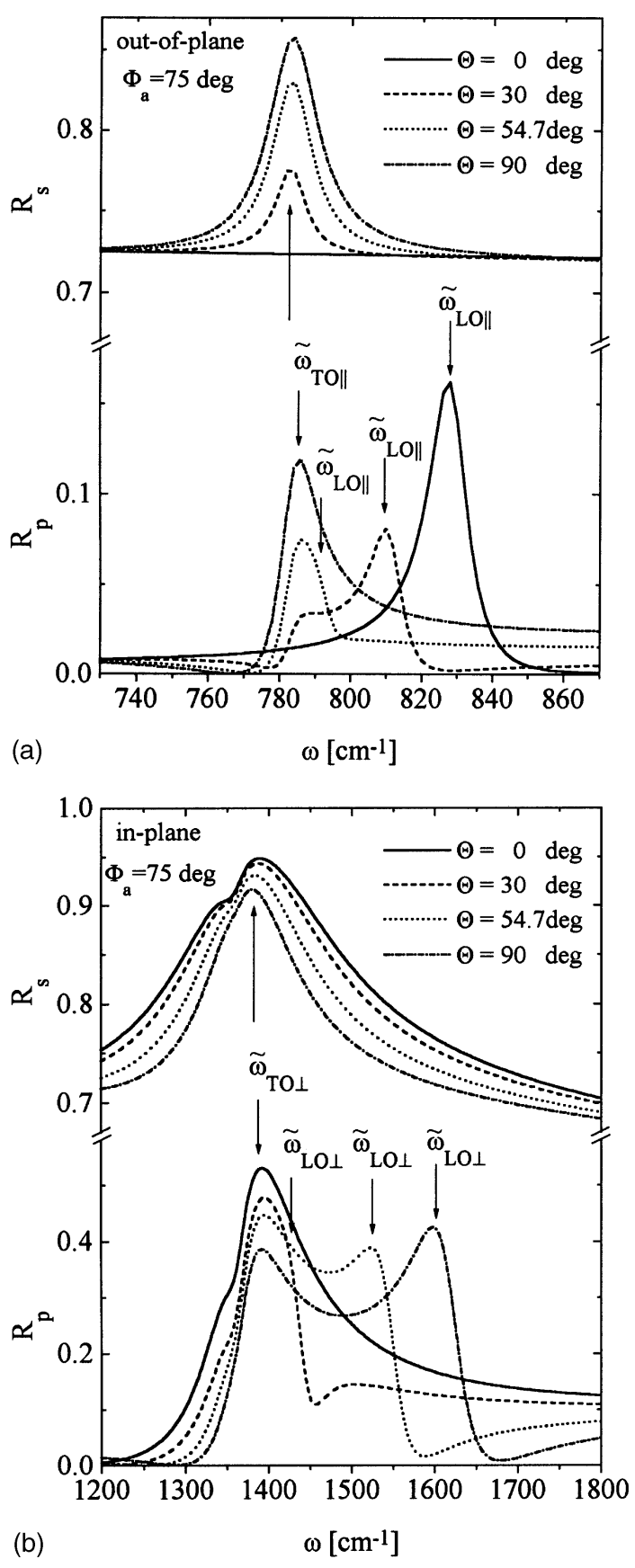

FIG. 3. (a) Simulated $R_{p}$ and $R_{s}$ of a 300-nm-thick $h$-BN thin film on silicon, vs the wave number for different grain $c$-axis orientations $\Theta$ within the $h$-BN out-of-plane resonance spectral region $\left(\Phi_{a}=75^{\circ} ; h\right.$-BN bulk data as used in Fig. 2 and given in Table I: $\Gamma_{\|}=40 \mathrm{~cm}^{-1}$ and $\left.\Gamma_{\perp}=60 \mathrm{~cm}^{-1}\right)$. The $p$-polarized wave components couple into the LO mode $\widetilde{\omega}_{\mathrm{LO} \|}$, which by itself is a function of $\Theta$. The $s$-polarized wave components excite only $\widetilde{\omega}_{\mathrm{TO} \|}$ (Berreman effect; Ref. 23). The TO mode does not depend on $\Theta$, whereas $\widetilde{\omega}_{\text {LO\|l }}$ shifts to lower wave numbers as $\Theta$ tends to $90^{\circ}$. The LO mode merges with $\widetilde{\omega}_{\mathrm{TO} \|}$, and cannot be excited at $\Theta=90^{\circ}$. (b) Same as (a) for the $h$-BN in-plane resonance spectral region. Here $\widetilde{\omega}_{\mathrm{LO} \perp}$ merges with $\widetilde{\omega}_{\mathrm{TO} \perp}$ as $\Theta$ tends to $0^{\circ}$, where $\widetilde{\omega_{\mathrm{LO} \perp}}$ finally disappears. Except for $\Theta=0^{\circ}$ both TO modes $\widetilde{\omega}_{\mathrm{TO} \|}$ [Fig. 3(a)] and $\widetilde{\omega}_{\mathrm{TO} \perp}$ are observable. At $\Theta=0^{\circ}$, the incident electric-field components excite only $\widetilde{\omega}_{\mathrm{LO} \|}$ [see Fig. 3(a)] and ${\widetilde{\omega_{\mathrm{TO}}}}_{\perp}$, representing an unusual case where the thin-film LO mode is at smaller wave numbers than the thin-film TO mode. 
TABLE I. Results from the best $c$-BN and $h$-BN thin-film lattice resonance parameters and sample structures obtained in this work.

\begin{tabular}{|c|c|c|c|c|c|c|c|c|c|c|c|c|}
\hline $\begin{array}{l}\text { Sample } \\
U_{B} /(\mathrm{V})\end{array}$ & $\mathrm{BN}$ & Layer & $\begin{array}{c}d \\
(\mathrm{~nm})\end{array}$ & $\begin{array}{l}\Theta \\
\left(^{\circ}\right)\end{array}$ & $\begin{array}{c}f_{c-\mathrm{BN}} \\
(\%)\end{array}$ & $\begin{array}{c}E \| c \\
\omega_{\mathrm{TO}} \| \\
\left(\mathrm{cm}^{-1}\right)\end{array}$ & $\begin{array}{c}\omega_{\mathrm{LO} \|} \\
\left(\mathrm{cm}^{-1}\right)\end{array}$ & $\begin{array}{c}\Gamma_{\|} \\
\left(\mathrm{cm}^{-1}\right)\end{array}$ & $\begin{array}{c}E \perp c \\
\omega_{\mathrm{TO} \perp} \\
\left(\mathrm{cm}^{-1}\right)\end{array}$ & $\begin{array}{c}\omega_{\mathrm{LO} \perp} \\
\left(\mathrm{cm}^{-1}\right)\end{array}$ & $\begin{array}{c}\Gamma_{\perp} \\
\left(\mathrm{cm}^{-1}\right)\end{array}$ & $\varepsilon_{0}{ }^{\mathrm{b}}$ \\
\hline$c \mathrm{BN} 1$ & $c / h^{\mathrm{a}}$ & 2 & 120 & $* \mathrm{a}$ & 75 & & & & $1070^{\mathrm{c}}$ & $1300^{c}$ & $130^{\mathrm{c}}$ & 5.5 \\
\hline-150 & $h$ & 1 & 5 & $\sim 90$ & & 787 & 819 & 21 & 1374 & 1610 & 69.5 & $\langle 4.2\rangle$ \\
\hline$c \mathrm{BN} 2$ & $c / h^{\mathrm{a}}$ & 2 & 131 & $* \mathrm{a}$ & 70 & & & & $1070^{c}$ & $1300^{c}$ & $130^{\mathrm{c}}$ & 5.5 \\
\hline-150 & $h$ & 1 & 27 & $\sim 70$ & & 782 & 819 & 24 & 1385 & 1610 & 79 & $\langle 4.7\rangle$ \\
\hline$h \mathrm{BN} 1$ & $h$ & 2 & 178 & $\sim 35$ & & 766 & 811 & 28 & 1400 & 1586 & 143 & $\langle 3.2\rangle$ \\
\hline$+20^{\mathrm{d}}$ & $h$ & 1 & 100 & $\sim 55$ & & & & 80 & & & 63.5 & $\langle 4.1\rangle$ \\
\hline $\begin{array}{l}h \mathrm{BN} 2 \\
-30 \mathrm{~V}\end{array}$ & $h$ & 1 & 520 & $\sim 90$ & & 773 & 820 & 60 & 1406 & 1579 & 130 & $\langle 3.9\rangle$ \\
\hline Ref. 14 & bulk $h$ & & & & & 783 & 828 & 8 & 1367 & 1610 & 29 & $7_{\|}, 5_{\perp}$ \\
\hline Ref. 16 & bulk $c$ & & & & & & & & $1056^{\mathrm{c}}$ & $1305^{\mathrm{c}}$ & $62^{c}$ & 6.8 \\
\hline
\end{tabular}

${ }^{a}$ EMA $\left(c\right.$-BN and isotropic $h$-BN, $\left.\Theta=54.7^{\circ}\right) . h$-BN parameters are the same as in layer 1.

${ }^{\mathrm{b}} h-\mathrm{BN}:\left\langle\varepsilon_{0}\right\rangle=\varepsilon_{0 \|} / 3+2 \varepsilon_{0 \perp} / 3$.

${ }^{\mathrm{c}} \mathrm{C}$-BN lattice absorption parameters.

${ }^{\mathrm{d}}$ Floating potential; no supplied substrate bias voltage.

information of both $R_{p}$ and $R_{s}$. To clarify the $h$-BN thinfilm ellipsometric reflectivity behavior, Fig. 4 shows simulations of $\Psi$ and $\Delta$ spectra. We used all model parameter as in Figs. 3(a) and 3(b), except the incidence angle which is now $65^{\circ}$. It should be pointed out that $R_{p}$ and $R_{s}$ depend on the incidence angle, i.e., the absolute values change as $\Phi_{a}$ varies. However, at oblique angle of incidence the TO and LO modes are excited at the same wave numbers as discussed above. Note that the relative change in the $p$-polarized reflectance due to the LO-phonon resonance absorption is positive (or negative) for plane waves incident at an angle $\Phi_{a}$ below (or above) the Brewster angle, respectively.

The ellipsometric spectra are more difficult to interpret, since they represent a complex ratio, but the above outlined TO and LO properties are observable as well: The pronounced peaks in $\Psi$ at $\omega=\widetilde{\omega}_{\mathrm{TO} \|}$ and $\omega=\widetilde{\omega}_{\mathrm{TO} \perp}$ are the $\mathrm{TO}$ resonant frequencies. The minima in $\Psi$ are due to the loss of $p$-polarized reflectance for incident light wave frequencies above the LO mode frequencies [see also Figs. 3(a) and 3(b)], and shift as a function of $\Theta$. Except at $\Theta=90^{\circ}$ the in-plane LO mode excitation causes a slope change in $\Psi$ and $\Delta$. At $\omega \sim \widetilde{\omega}_{\mathrm{LO} \|}$ and $\Theta=90^{\circ}$ the signal in $\Psi$ and $\Delta$ is reversed due to the LO resonance excitation, i.e., $R_{s}$ is constant, and $R_{p}$ changes due to the LO-phonon absorption [see Fig. 3(a)]. At this point it is emphasized that the simulations shown in Figs. 3(a), 3(b), and 4 depend on the resonance broadening parameters, in that the TO and LO modes become very pronounced for nondissipative lattice vibrations, or smear out for large broadening parameters. The $\Psi$ and $\Delta$ spectra need to be analyzed using a detailed line shape modeling of the thin-film dielectric function presented in Eqs. (2a) and (2b). Note also the asymmetric broadening of $\Psi$ within the in-plane resonance region caused by the asymmetric $R_{p}$ and $R_{s}$ line shape shown in Fig. 3(b). Note finally that $\cos \Theta=1 / \sqrt{3}$ represents the case of completely averaged $c$-axis orientations of the $h$-BN grains (isotropically averaged $h$-BN). The isotropic reflectivity behavior is shown in Figs. 3(a), 3(b), and 4 by the dotted lines labeled by $\Theta$ $=54.7^{\circ}$.

\section{B. $c$-BN dielectric function model}

Bulk $c$-BN is optically isotropic $(a=3.6157 \AA) .{ }^{19}$ Recent microscopic investigations demonstrate the existence of the $h$-BN modification at the $c$-BN grain boundaries within the high-content $c$-BN thin films. ${ }^{4}$ Our HRTEM results did not indicate the existence of long-range ordered $h$-BN in our samples. We therefore treat the grain boundary material in $c$-BN as isotropically averaged $h$-BN.

If a layer consists of a mixture of two isotropic materials $a$ and $b$, with the microstructural dimensions much less than a fraction of the wavelength of light, then the macroscopic dielectric function can be described with the Bruggeman effective-medium approximation (EMA)

$$
f_{a} \frac{\varepsilon_{a}-\varepsilon}{\varepsilon_{a}+2 \varepsilon}+f_{b} \frac{\varepsilon_{b}-\varepsilon}{\varepsilon_{b}+2 \varepsilon}=0,
$$

where $\varepsilon_{a}, \varepsilon_{b}, f_{a}$, and $f_{b}$ are the dielectric functions and volume fractions of materials $a$ and $b$, respectively. ${ }^{24}$

Equation (1) was used to model the isotropic dielectric function of $c$-BN, whereas Eq. (2b) at $\cos ^{2} \Theta=\frac{1}{3}$ describes $\varepsilon_{h}=\varepsilon_{o, \text { eff }}=\varepsilon_{e, \text { eff }}$ for isotropic $h$-BN. Equation (6) was then used to model the dielectric response $\varepsilon$ of a layer which contains $c$-BN and randomly oriented $h$-BN. Substituting $f_{b}=\left(1-f_{a}\right)$ the average composition parameter $f_{a}=f_{c-\mathrm{BN}}$ can be used to estimate the volume fraction of $c-\mathrm{BN}$ within the high $c$-BN content layer.

\section{Sample analysis}

The results of ellipsometric measurements are expressed in terms of $\Psi$ and $\Delta$. In general, and if the sample under consideration contains multiple layers, or at least one layer, the spectroscopic data must be numerically fit. For this purpose, a model must be assumed, and $\Psi$ and $\Delta$ are generated and compared with the measured data for the same wave number and incidence set. ${ }^{13} \Psi$ and $\Delta$ depend then on a set of parameters, i.e., the dielectric function and thickness for each layer. The model should include all necessary characteristics 


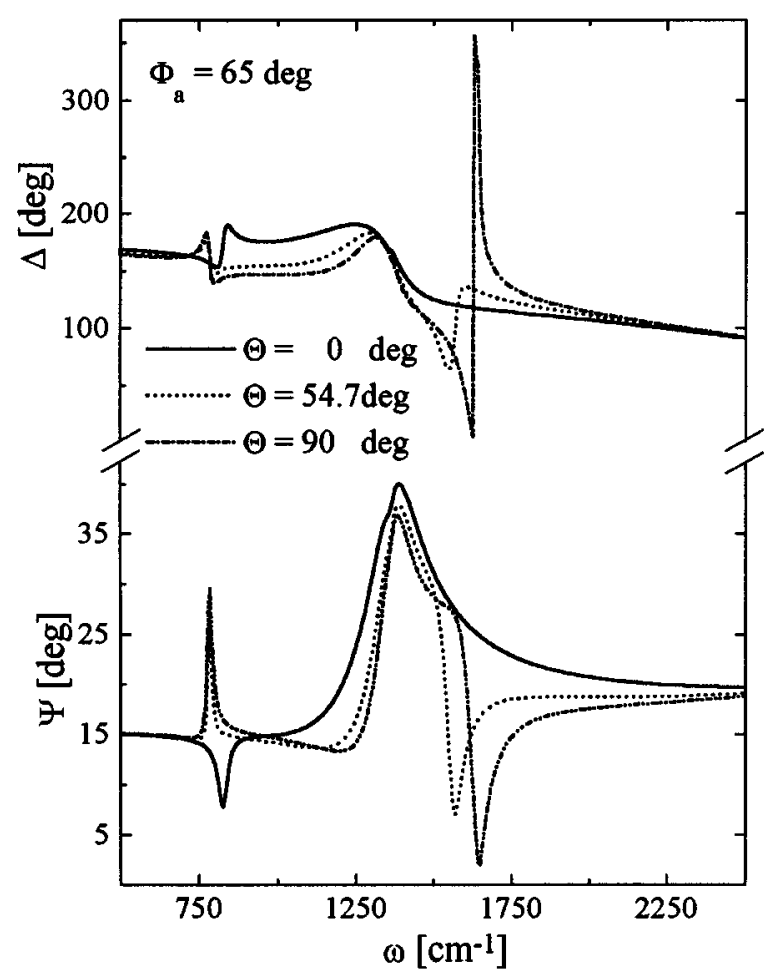

FIG. 4. Same as Figs. 3(a) and 3(b) for $\Psi$ and $\Delta$ at $\Phi_{a}=65^{\circ}$. Similar spectral dependencies are observable through the complex reflectance ratio in terms of $\Psi$ and $\Delta$ as described for $R_{p}$ and $R_{s}$ in Figs. 3(a) and 3(b). The pronounced peaks in $\Psi$ are due to the TO modes. At $\Theta=0^{\circ}$ the signal in $\Psi$ within the out-of-plane resonance spectral region is reversed due to the absence of the TO mode and domination of the LO mode. The in-plane LO mode signal at $\omega$ $=\widetilde{\omega}_{\mathrm{LO} \perp}$ is merged within both $\Psi$ and $\Delta$ spectra, and a slope change appears instead. However, the shift of the extreme in $\Psi$ and $\Delta$ above $\widetilde{\omega}_{\mathrm{LO} \perp}$ is a direct indication for the $\Theta$ dependence of the LO modes.

to simulate the behavior of the actual sample realistically. A regression analysis was used to vary the model parameters, such as the $c$-BN and $h$-BN lattice resonance parameters, until the generated and measured values matched as close as possible. This was done by minimizing the weighted meansquare error function which is the sum over the squared differences between the measured and calculated $\Psi$ and $\Delta$ values divided by the measurement uncertainty.

Figures 5(a) and 5(b) show experimental and generated data $(\Psi$ and $\Delta)$ obtained on two different $h$-BN samples $(h \mathrm{BN} 1, h \mathrm{BN} 2)$. The sample growth parameters (i.e., the substrate bias voltage $U_{B}$ ) are given in Table I. Data from one angle of incidence are presented only to avoid confusion between the otherwise multiple plots. However, the results and conclusions to be drawn through the following discussions are valid for all incidence angle data. According to HRTEM and SAD investigations, two different models were established. A single anisotropic layer was used to represent the optical response of sample $h \mathrm{BN} 2$, whereas a two-layer model (bottom layer: isotropic averaged $h$-BN; top layer: anisotropic $h$-BN) was applied to predict $\Psi$ and $\Delta$ obtained from sample $h \mathrm{BN} 1$. The generated data plotted in Figs. 5(a) and 5(b) refer to the best fit (each sample separately) for the layer thicknesses, the cone angles $\Theta$, and the in-plane and
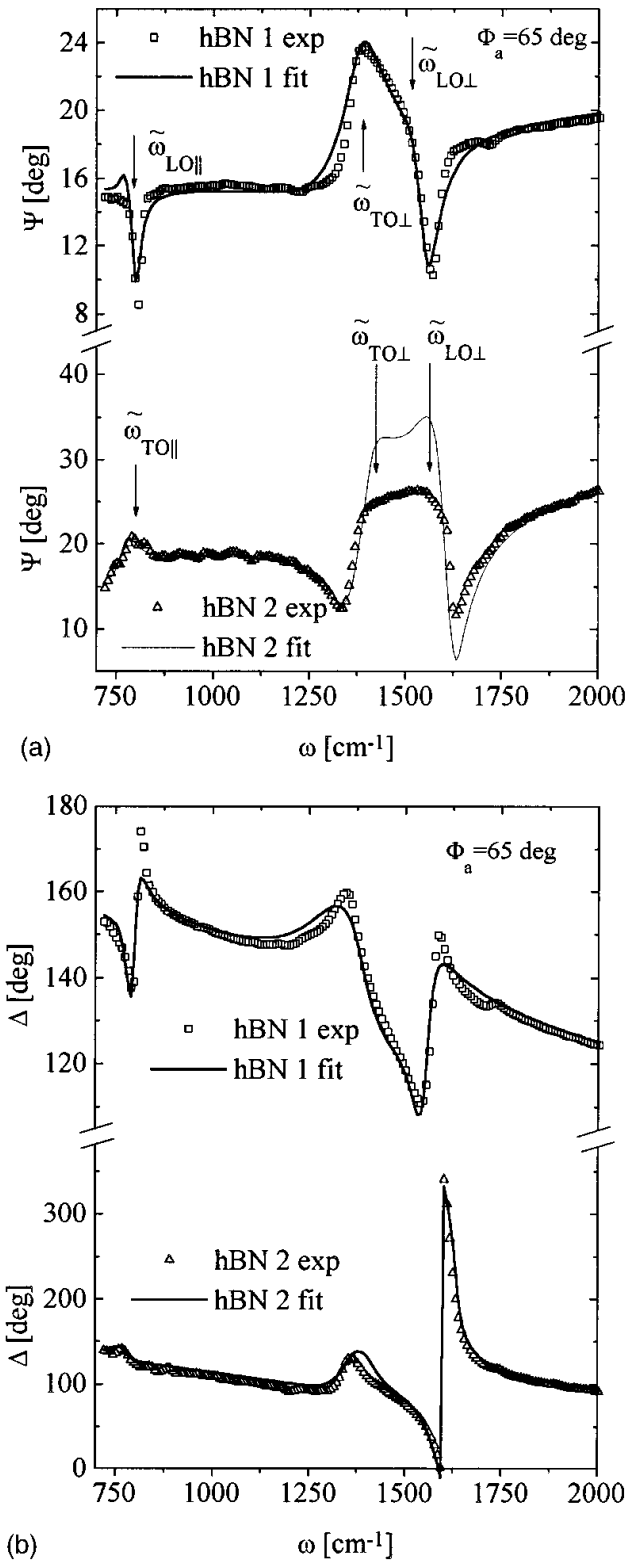

FIG. 5. (a) Experimental and generated data (solid lines) obtained from the $h$-BN thin-film samples ( $\Psi$ only; $\Phi_{a}=65^{\circ} ; h \mathrm{BN} 1$ : open squares; $h \mathrm{BN} 2$ : open triangles). The generated data were taken from the best fit for $\omega_{\mathrm{TO} \|}, \omega_{\mathrm{LO} \|}, \varepsilon_{0 \|}, \Gamma_{\|}, \omega_{\mathrm{TO} \perp}, \omega_{\mathrm{LO} \perp}$, $\varepsilon_{0 \perp}$, and $\Gamma_{\perp}$, the average grain $c$-axis orientations $\Theta$, and the particular layer thicknesses. The out-of-plane TO resonance is difficult to observe in sample $h \mathrm{BN} 1\left(\Theta=35^{\circ}\right)$. The LO mode appears instead. As well, the in-plane LO mode is shifted toward $\widetilde{\omega}_{\mathrm{TO} \perp}$. Sample $h \mathrm{BN} 2\left(\Theta=90^{\circ}\right)$ reveals $\widetilde{\omega}_{\mathrm{TO} \|}$ instead of $\widetilde{\omega}_{\mathrm{LO} \|}$. The inplane LO mode appears at higher wave numbers. Both samples, however, possess the same in-plane TO modes. (b) Same as (a) for $\Delta$. The $2 \pi$ sign flip in $\Delta$ measured on sample $h \mathrm{BN} 2$ indicates the pronounced LO mode $\widetilde{\omega}_{\mathrm{LO} \perp}$ as demonstrated in Fig. 4. [The data in this figure are reprinted (abstracted) with permission from Franke et al., Appl. Phys. Lett. 70, 1668 (1997). Copyright 1997 AIP.]

out-of-plane lattice resonance parameters $\varepsilon_{0 \perp}, \omega_{\mathrm{TO} \perp}$, $\omega_{\mathrm{LO} \perp}$, and $\Gamma_{\perp}$, and $\varepsilon_{0 \|}, \omega_{\mathrm{TO} \|}, \omega_{\mathrm{LO} \|}$, and $\Gamma_{\|}$, respectively. Within the two-layer model the lattice resonance parameters were assumed to be the same for both layers. Only the broadening parameters were allowed to vary independently to ac- 
count for different grain sizes or packing densities. The results are summarized in Table I. The last column displays the average static dielectric constant $\left\langle\varepsilon_{0}\right\rangle=\varepsilon_{0 \|} / 3+2 \varepsilon_{0 \perp} / 3$. Due to their different microstructures both samples show the above discussed anisotropic reflectivity: sample $h \mathrm{BN} 1$ contains both grains with their $c$ axes bent by approximately $35^{\circ}$ from the sample normal as well as grains with randomly oriented (isotropic) $h$-BN. A strong absorption due to the $\widetilde{\omega}_{\mathrm{TO} \perp}$ frequency common to both layers is seen. The LO mode frequency $\widetilde{\omega}_{\mathrm{LO} \perp}$ is excited within the $h$-BN bottomlayer, whereas the $\widetilde{\omega}_{\mathrm{LO} \|}$ frequency causes resonance absorption in the top-layer only [arrows in Fig. 5(a)]. Sample $h$ BN2 was modeled by a single layer where the average $h$-BN grain $c$-axis orientation was found parallel to the sample surface $\left(\Theta \sim 90^{\circ}\right)$. Here the out-of-plane TO mode resonance at $\widetilde{\omega}_{\mathrm{TO} \|}$ is observable, and the LO mode resonance $\widetilde{\omega}_{\mathrm{LO} \|}$ is absent. The in-plane TO mode $\left(\widetilde{\omega}_{\mathrm{TO} \perp}\right)$ is the same as $h \mathrm{BN} 1$, and the more pronounced LO mode at $\widetilde{\omega}_{\mathrm{LO} \|}$ is shifted to higher wave numbers, in agreement with the above outlined theoretical considerations. Note the $2 \pi$ polarity flip in $\Delta$ [Fig. 5(b)], which indicates the most separated LO mode $\widetilde{\omega}_{\mathrm{LO} \perp}$ for $\Theta=90^{\circ}$, as demonstrated through the simulations of $\Psi$ and $\Delta$ in Fig. 4. Note also the spectral shift between the in-plane LO mode resonance excited in the top-layer in $h \mathrm{BN} 1\left(\Theta \sim 35^{\circ}\right)$ and in the single layer in $h \mathrm{BN} 2(\Theta$ $\sim 90^{\circ}$ ).

The differences between the generated and measured $\Psi$ data from sample $h \mathrm{BN} 2$ within the in-plane mode spectral region may be due to a certain amount of water within the film. The differences decrease by adding water as a third constituent within the dielectric function model. In addition, data taken immediately after annealing of the sample in vacuum differ from the values presented here to the extent that the generated data in Fig. 5(a) then match to the measured values. The annealing process is reversible, i.e., after some hours of sample exposure to air the ellipsometric parameters become identical to the data taken before sample annealing. Although the latter is indicative of an exchange process in which water could participate, we still seek for further experimental evidence to prove this hypothesis.

Figures 6(a) and 6(b) depict the same data for the highcontent $c$-BN samples $c$ BN1 and $c \mathrm{BN} 2$. Their structural and material properties are summarized in Table I as well. Here a two-layer model was used to account for the noncubic nucleation layer. The latter was included as an anisotropic $h$-BN layer. The isotropic high-content $c$-BN layer was simulated through the Bruggeman EMA approximation. As a result, the estimated phase composition $f_{c}$-BN was obtained from the best fit which included the layer thicknesses, the $c$-BN and $h$-BN lattice resonance parameters and cone angle $\Theta$ for the nucleation layer. The $h$-BN parameters within the bottom and top layers were again assumed to be unique, except for the cone angles and broadening parameters. The nucleation layers were found to be anisotropic, owing an average $c$-axis orientation nearly parallel to the sample surface. The particular shift of their LO mode frequencies $\widetilde{\omega}_{\mathrm{LO} \perp}$ gives rise to the small variations in $\Psi$ and $\Delta$, as indicated by arrows in Fig. 6(b): sample $c$ BN1 shows a small absorption due to the nucleation layer in-plane LO mode for $\Theta \sim 90^{\circ}$, which is
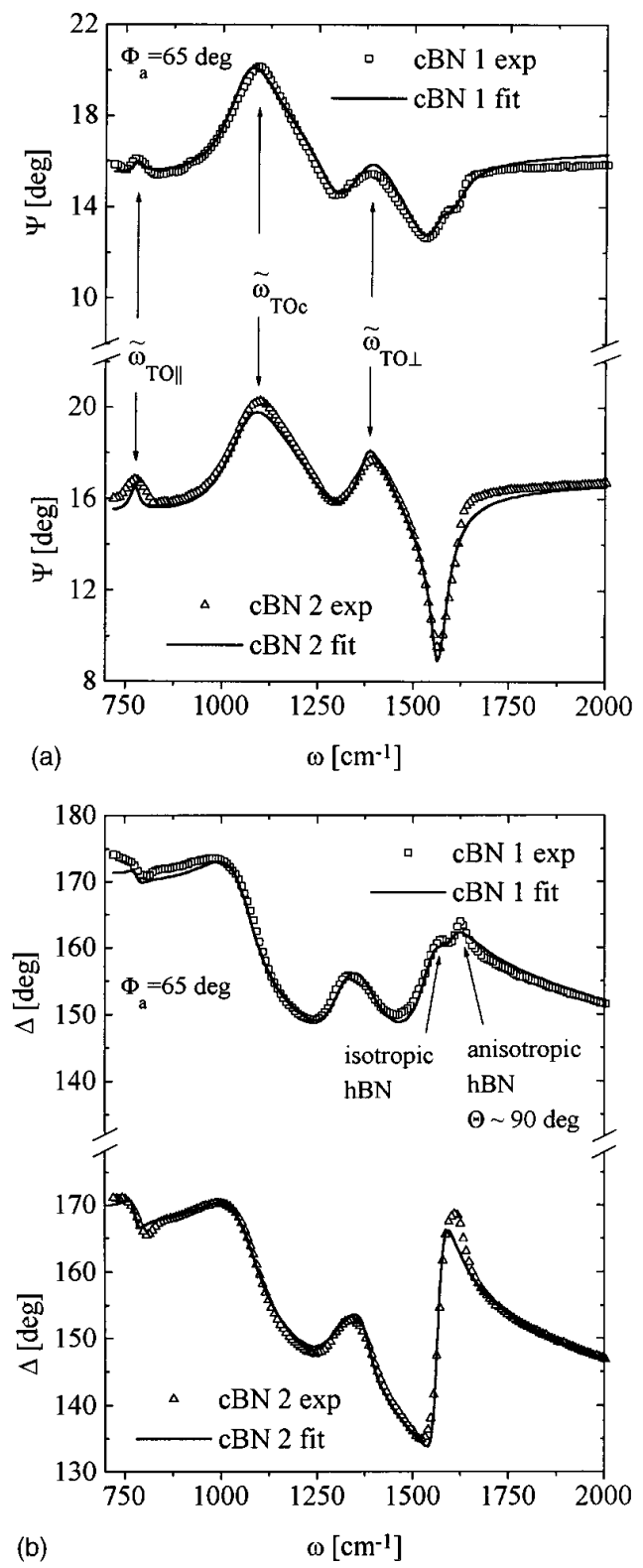

FIG. 6. (a) Same as Fig. 5(a) for the high-content $c$-BN thinfilm samples ( $c \mathrm{BN} 1$ : open squares; $c \mathrm{BN} 2$ : open triangles). The generated data (solid lines) were obtained from the best fit for the $c$-BN and $h$-BN dielectric function models as discussed in the text. The arrows indicate the strongly pronounced $c$-BN TO mode and the $h$-BN in-plane and out-of-plane TO modes. (b) Same as (a) for $\Delta$. The resonance structure at $\sim 1610 \mathrm{~cm}^{-1}(c \mathrm{BN} 1)$ is due to the LO modes $\widetilde{\omega}_{\mathrm{LO} \perp}\left(\right.$ at $\left.\Theta=54.7^{\circ}\right)$ of randomly oriented (isotropic) $h$-BN within the high-content $c$-BN layer and $\widetilde{\omega}_{\mathrm{LO} \perp}\left(\right.$ at $\left.\Theta \sim 90^{\circ}\right)$ of the anisotropic nucleation layer. The same structure does not occur in sample $c \mathrm{BN} 2$. Both modes merge and intensify since $\widetilde{\omega}_{\mathrm{LO} \perp}(\Theta$ $\left.\sim 70^{\circ}\right)$ tends to $\widetilde{\omega}_{\mathrm{LO} \perp}\left(\Theta=54.7^{\circ}\right)$. [The data in this figure are reprinted (abstracted) with permission from Franke et al., Appl. Phys. Lett. 70, 1668 (1997). Copyright 1997 AIP.]

spectrally separated from the isotropic $h$-BN LO mode $(\Theta$ $=54.7^{\circ}$ ). Both modes merge and intensify in sample $c \mathrm{BN} 2$, where the nucleation layer $c$-axis grain orientation was found to be approximately $70^{\circ}$. The pronounced $c$ - $\mathrm{BN}$ TO mode 
$\left(\widetilde{\omega}_{\mathrm{TO} c}\right)$ observable now in $\Psi$ and $\Delta$ is indicated by arrows, together with the $h$-BN TO mode frequencies $\widetilde{\omega}_{\mathrm{TO} \perp}$ and $\widetilde{\omega}_{\mathrm{TO} \|}$ which still appear.

The microstructure parameters obtained from our ellipsometric model are consistent with HRTEM and SAD investigations. The resulting $c$-BN optical lattice properties are in good agreement with the bulk data reported by Eremets et al. (Ref. 16). The LO and TO mode frequencies observed on our $h$-BN thin films differ slightly from the bulk modes obtained by Geick, Perry, and Rupprecht (Ref. 14). The latter is regarded as being due to the more complicated microstructuredependent thin-film reflectivity behavior for which our rather ideal model was assumed to be valid. We have not accounted for a variation in grain size, nor as well as a larger range of possible $c$-axis grain orientations instead of those mapped onto a single cone. However, we were able to explain different polarization-dependent reflectivity data obtained from $h$-BN samples having unique chemical composition. The present analysis approach can be extended to other anisotropic dielectric function models for more generally distributed polycrystalline materials.

\section{SUMMARY}

We have presented a microstructure-dependent anisotropic dielectric function model for polycrystalline hexagonal boron nitride from which we have derived and discussed the transverse- and longitudinal-optical modes of thin films. We describe an effective-medium approach for infrared optical properties of phase compositions of $c-\mathrm{BN}$ and randomly oriented (isotropic) $h$-BN material. Infrared ellipsometry over the wavelength range from 700 to $3000 \mathrm{~cm}^{-1}$ was then used to determine the phase and microstructure of polycrystalline hexagonal and cubic boron nitride thin films. The ellipsometric data revealed the layer-phase composition and the average orientation of the hexagonal grain $c$ axes. The hexagonal boron nitride $s p^{2}$-bonded basal plane orientations were found to vary depending on the growth conditions. The phase composition of the high $c$-BN content layers were obtained from the infrared dielectric function using an effective-medium approximation. The BN nucleation layer consist of anisotropic $h$-BN with a preferred grain $c$-axis orientation parallel to the thin-film interface.

The results obtained demonstrate the capabilities of infrared spectroscopic ellipsometry to perform detailed investigations of phase and microstructure of mixed-phase samples such as BN thin films. The method described here can be used as a standard tool to access sample information similar to transmission electron microscopy investigations without complicated sample preparation techniques. The knowledge of phase and microstructure is essential for a better understanding of the thin-film growth mechanism and properties of the mixed-phase thin films.

\section{ACKNOWLEDGMENTS}

We wish to acknowledge G. Wagner at the University of Leipzig and M. Röder at the Technical University ChemnitzZwickau for SAD and HRTEM investigations. Further, we thank Daniel W. Thompson at the University of NebraskaLincoln for valuable discussions and technical guidance during the infrared ellipsometric investigations. We thank the Center for Microelectronic and Optical Materials Research of the University of Nebraska-Lincoln for financial support during part of the work.
*Electronic address: mschub@physik.uni-leipzig.de

${ }^{1}$ T. A. Friedmann, P. B. Mirkarimi, D. L. Medlin, K. F. McCarty, E. J. Klaus, D. R. Boehme, H. A. Johnson, M. J. Mills, and D. K. Ottesen, J. Appl. Phys. 76, 3088 (1994).

${ }^{2}$ H. Hofsäss, C. Ronning, U. Griesmeier, M. Gross, S. Reinke, and M. Kuhr, Appl. Phys. Lett. 67, 46 (1995).

${ }^{3}$ S. Reinke, M. Kuhr, W. Kulisch, and R. Kassing, Diamond Relat. Mater. 4, 272 (1995).

${ }^{4}$ W. L. Zhou, Y. Ikuhara, M. Murakawa, S. Watanabe, and T. Suzuki, Appl. Phys. Lett. 66, 2490 (1995).

${ }^{5}$ D. J. Kester, K. S. Ailey, D. J. Lichtenwalner, and R. F. Davis, J. Vac. Sci. Technol. A 12, 3074 (1994).

${ }^{6}$ D. L. Medlin, T. A. Friedmann, P. B. Mirkarimi, P. Rez, M. J. Mills, and K. F. McCarty, J. Appl. Phys. 76, 295 (1994).

${ }^{7}$ M. Schubert, B. Rheinländer, E. Franke, H. Neumann, J. Hahn, M. Röder, and F. Richter, Appl. Phys. Lett. 70, 1819 (1997).

${ }^{8}$ A. S. Rozenberg, Yu. A. Sinenko, and N. V. Chukanov, J. Mater. Sci. 28, 5675 (1993).

${ }^{9}$ T. Kuzuba, K. Era, T. Ishii, and T. Sato, Solid State Commun. 25, 863 (1978).

${ }^{10}$ D. M. Hoffman, G. L. Doll, and P. C. Eklund, Phys. Rev. 30, 6051 (1984).

${ }^{11}$ M. F. Plass, W. Fukarek, S. Mändl, and W. Möller, Appl. Phys. Lett. 69, 46 (1996).
${ }^{12}$ E. Franke, H. Neumann, M. Schubert, T. E. Tiwald, J. A. Woollam, J. Hahn, and F. Richter, Appl. Phys. Lett. 70, 1668 (1997).

${ }^{13}$ R. M. A. Azzam and N. M. Bashara, Ellipsometry and Polarized Light (North-Holland, Amsterdam, 1977).

${ }^{14}$ R. Geick, C. H. Perry, and G. Rupprecht, Phys. Rev. 146, 543 (1966).

${ }^{15}$ P. J. Gielisse, S. S. Mitra, J. N. Plendl, R. D. Griffis, L. C. Mannsur, R. Marshall, and E. A. Pascoe, Phys. Rev. 155, 1039 (1967).

${ }^{16}$ M. I. Eremets, M. Gauthier, A. Polian, J. C. Chervin, J. M. Besson, G. A. Dubitskii, and Ye. Yc. Semenova, Phys. Rev. B 52, 8854 (1995).

${ }^{17}$ J. Hahn, M. Friedrich, R. Pintaske, M. Schaller, N. Kahl, D. R. T. Zahn, and F. Richter, Diamond Relat. Mater. 5, 1103 (1996).

${ }^{18}$ M. Röder, J. Hahn, U. Falke, S. Schulze, F. Richter, and M. Hietschold, Michrochim. Acta 129, 283 (1997).

${ }^{19}$ R. Poerschke and O. Madelung, Semiconductors Group IV Elements and III-V Compounds (Springer, Berlin, 1991).

${ }^{20}$ M. Schubert, Phys. Rev. B 53, 4265 (1996).

${ }^{21}$ C. Kittel, Introduction to Solid State Physics (Wiley, New York, 1976).

${ }^{22}$ M. Balkanski, in Handbook on Semiconductors (North-Holland, New York, 1982), Vol. II.

${ }^{23}$ D. W. Berreman, Phys. Rev. 130, 2193 (1963).

${ }^{24}$ D. E. Aspnes and J. B. Theeten, Phys. Rev. B 20, 3292 (1979). 